\title{
RELAY FEEDBACK AUTOTUNing - IMPLEMENTATION AND SIMULATION
}

\author{
Roman Prokop \& Jiří Korbel
}
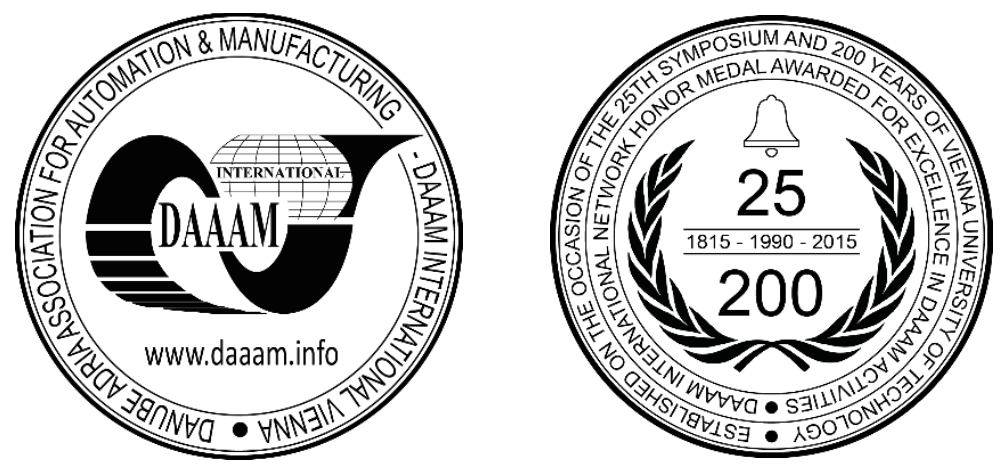

This Publication has to be referred as: Prokop, R[oman] \& Korbel, J[iri] (2018). Relay Feedback Autotuning Implementation and Simulation, Proceedings of the 29th DAAAM International Symposium, pp.0137-0143, B. Katalinic (Ed.), Published by DAAAM International, ISBN 978-3-902734-20-4, ISSN 1726-9679, Vienna, Austria DOI: $10.2507 / 29$ th.daaam.proceedings.019

\begin{abstract}
The paper brings some authors' results in the field of autotuning research. Autotuners represent a combination of a relay feedback experiment and a suitable control design method. The contribution deals with SISO systems where symmetrical or asymmetrical relay can be used. Asymmetric relay with hysteresis feedback experiments enable to estimate three parameters for aperiodic first or second order transfer functions. After the identification experiment, controller parameters are derived through a linear diophantine equation in two different rings. The first ring is a set of proper and stable rational functions $R_{P S}$. The second ring $R_{M S}$ represents a set of RQ-meromorphic functions suitable for delay systems. Moreover, a final controller can be tuned by a scalar real parameter $m>0$. Many of designed controllers have the PI or PID form. A Matlab-Simulink program implementation was developed for simulation and verification of the studied approach. The emphasis is laid on the user-friendly program system for control design and simulation. Naturally, also higher order systems can be controlled by the approach. Illustrative examples support simplicity and efficiency of the proposed methodology in simulation and real-time control.
\end{abstract}

Keywords: auto-tuning; relay feedback; diophantine equation; PID controller

\section{Introduction}

The development of various autotuning principles was started by a simple symmetrical relay feedback experiment proposed by Åström and Hägglund [1], [2] and the scheme is depicted in Fig. 1. The ultimate gain and ultimate frequency are then used for adjusting of parameters by the original Ziegler-Nichols rules [2], [3], [13], for aperiodic tuning in [4] or [18]. The idea has gained wide popularity with control engineers and nowadays, many industrial controllers are equipped with automatic tuning abilities. During the period of three decades, many studies have been reported to extend and improve autotuners principles, see e.g. [5], [6], [8], [9].

In this paper, new combinations for autotunig methods of PI and PID controllers are proposed and developed. Theory of the algebraic approach is studied in [19]. Here, all autotuning principles combine an asymmetrical relay identification test and a control design procedure performed in the ring of $R_{P S}$ or $R_{M S}$. 
The pole placement problem in $R_{P S}$ ring is formulated through a Diophantine equation and the pole is analytically tuned according to aperiodic response of the closed loop. Further details and aspects can be found in [16], [18], [20]. Naturally, there exist many principles of control design syntheses that can be used for autotuning principles, see [24] as a monograph.

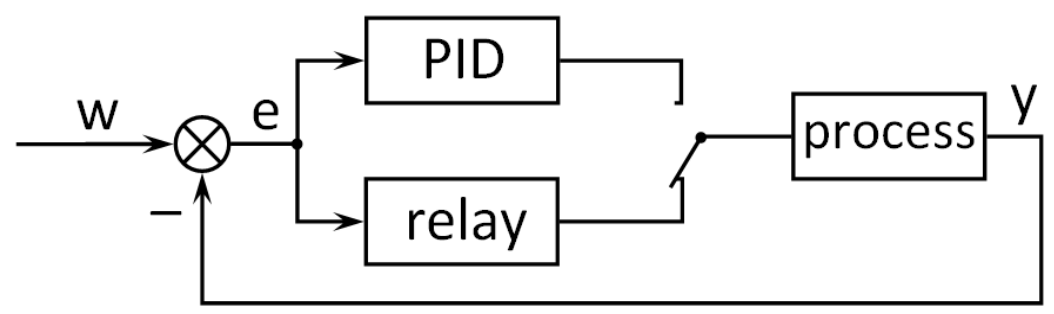

Fig. 1. Block diagram of an autotuning principle

\section{Relay estimation}

According to Fig. 1. in the relay feedback experiment various types of relay can be used. Originally, the symmetrical relay was used in [1], [2]. Later, the extension in relay utilization was performed in [5], [6], [9], [10], [11], [12], [15], [22] by an asymmetry and hysteresis of a relay. System instability is dealt in [11] while time-delayed systems are solved in [10], aperiodic or robust features are outlined in [12], [18]. Experiments with asymmetrical and dead-zone relay feedback are reported in [14], [15].

In the contribution, two simplest SISO linear dynamic systems with a delay term are used for relay approximation of the controlled plant. The first order (stable) plus dead time (FOPDT) model and the second order one plus dead time (SOPDT) are described by the transfer functions:

$$
G_{1}(s)=\frac{K}{T s+1} \cdot e^{-\Theta s}, \quad G_{2}(s)=\frac{K}{(T s+1)^{2}} \cdot e^{-\Theta s}
$$

The formulas for the calculation of estimated parameters (1) are generally known and can be found e.g. [7], [8], [16], [17], various details for relay parameter identification are adopted from [23], [24].

\section{Outline of Algebraic Control Design}

Algebraic notions, modules and tools were found to be useful in modern control theory for decades. Polynomials modules were firstly used in describing of linear systems continuous as well as discrete-time ones. The algebraic approach has dominated especially in the input-output properties of systems. The diophantine equation in the ring of polynomials became a simple and powerful tool for the discrete control synthesis as continuous-time, multivariable and nonlinear systems.

Apart from the ring of polynomials $R_{P}$, there are several rings used for control synthesis, e.g. the ring of stable and proper rational function $R_{P S}$ (e.g. [17], [20]), the ring of stable and proper meromorphic functions $R_{M S}$, see [8], [19], [21].

Briefly speaking, a ring is a set equipped with two operations, addition and multiplication, that forms a (Abel) group with respect to addition and a semi group with respect to multiplication. It means that in the ring is not generally possible to divide. An element having a multiplicative inverse is called a unit. The ring is a commutative one if multiplication is commutative. If every non-zero element is a unit, the set is called a field. The Diophantine equation in a ring has the form

$$
A X+B Y=C
$$

where $A, B, C$ are known elements and $X, Y$ are unknown elements of given ring. Equation (2) is solvable if every common divisor $A$ and $B$ divides $C$. Without loss of generality, it can be supposed that $A, B$ are coprime. Equation (2) has then an infinite number of solutions given by

$$
\begin{aligned}
& X=X_{0}-A Z \\
& Y=Y_{0}+B Z
\end{aligned}
$$

where $X_{0}, Y_{0}$ are a particular solution of (2) and $Z$ is an arbitrary element of a given ring. The general two-degree of freedom (2DOF) control loop is shown in Fig. 2. The classical one-degree of freedom (1DOF) is simply obtained by putting $R(s)=Q(s)$. 


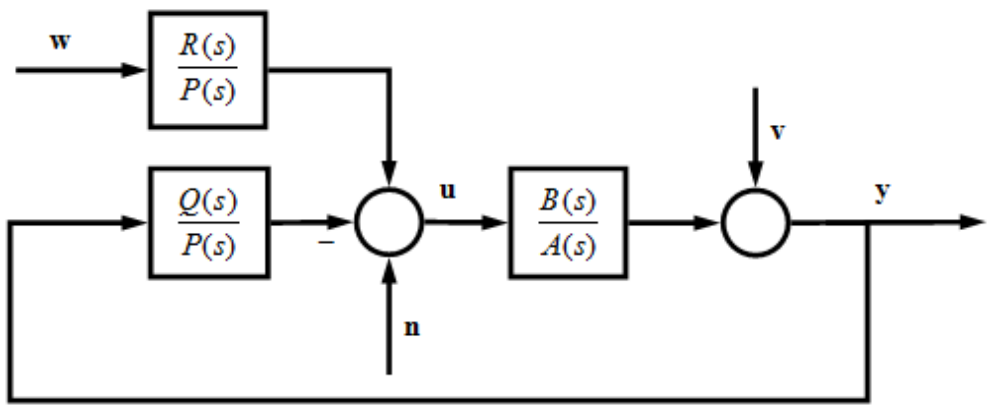

Fig. 2. Two-degree of freedom (2DOF) control loop

All feedback stabilizing controllers for the feedback system depicted in Fig. 1 are given by a general solution of the Diophantine equation:

$$
A P+B Q=1
$$

which can be expressed with $Z$ free in $R_{P S}$ :

$$
\frac{Q}{P}=\frac{Q_{0}-A Z}{P_{0}+B Z}
$$

The Diophantine equation for designing the feedforward controller depicted in Fig. 2 is:

$$
F_{w} S+B R=1
$$

with parametric solution again with $T$ free in $R_{P S}$ :

$$
\frac{R}{P}=\frac{R_{0}-F_{w} T}{P_{0}+B T}
$$

The derived formulas for both estimated systems with or without Pade approximation were published several times, they can be found e.g. [7], [8], [16], [19], [21]. The formulas are incorporated in the program system.

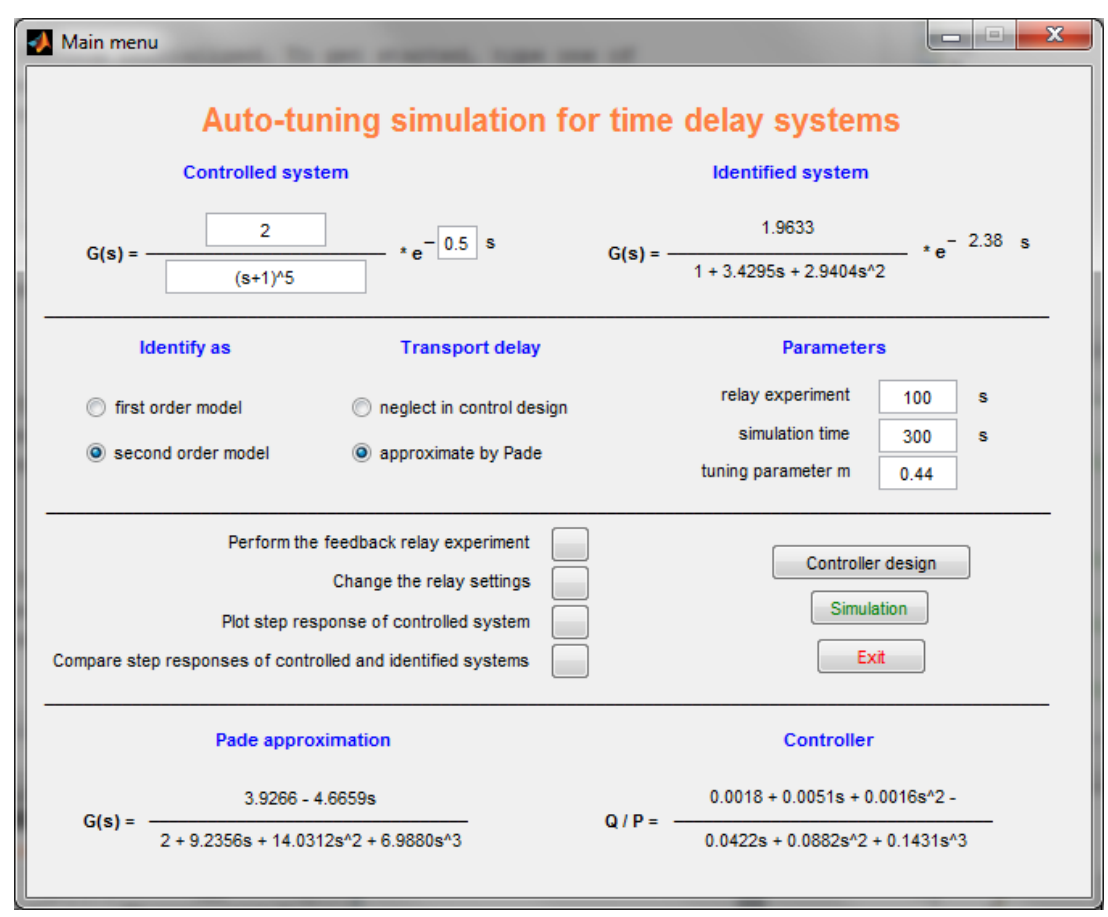

Fig. 3. Main Menu 


\section{Design Program System and Simulation}

A Matlab program system was developed for design, simulations and engineering utilization of auto-tuning principles. The program enables a choice for the estimation relay parameters of the controlled system of arbitrary order. The estimated model is of a first or second order transfer function without or with a time delay term. The user can choose two cases for time delay terms. The first case simply neglects the time term, in the second one the term is approximated by the Pade expansion. The program is developed with the support of the Polynomial Toolbox. The Main menu window of the program system can be seen in Fig. 3.

At the beginning, the controlled transfer function is defined and parameters for the relay experiment are adjusted. Then, the experiment is performed and it can be repeated with modified parameters if necessary. One of estimation experiment responses is depicted in Fig. 6. After the experiment, an estimated transfer function in the form of (1) performed automatically and controller parameters are generated after pushing of the appropriate button. The implicit value of tuning parameter $m>0$ is calculated according to [18] as an aperiodic one. Parameters for experimental adjustment are defined in the upper part of the window. Then, the second phase begins with the "Design controller parameters" button and the actual control design is performed. According to the above mentioned methodology and identified parameters, the controller is derived and displayed. The control scheme depends on the choice for the 1DOF or $2 \mathrm{DOF}$ structure and on the choice of the treatment with the time delay term. The last phase is a simulation one and the Simulink scheme according to Fig. 4 is performed.

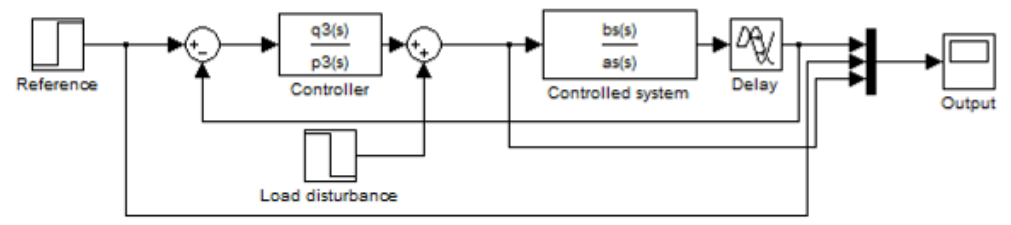

Fig. 4. Control simulation of 1DOF loop in Simulink

\section{Simulation and analysis}

The example represents a case of higher order system with a delay term. The transfer function $G(s)$ is supposed in the form:

$$
G(s)=\frac{2 e^{-0.5 s}}{(s+1)^{5}}
$$

The step responses of the original plant and FOPDT and SOPDT are shown in Fig. 5. The first and second order estimation obtained by a relay experiment gives the following transfer functions:

$$
\tilde{G}(s)=\frac{1.96}{3.39 s+1} \cdot e^{-2.96 s} \quad ; \quad \tilde{\tilde{G}}(s)=\frac{1.96}{2.94 s^{2}+3.43 s+1} \cdot e^{-2.38 s}
$$

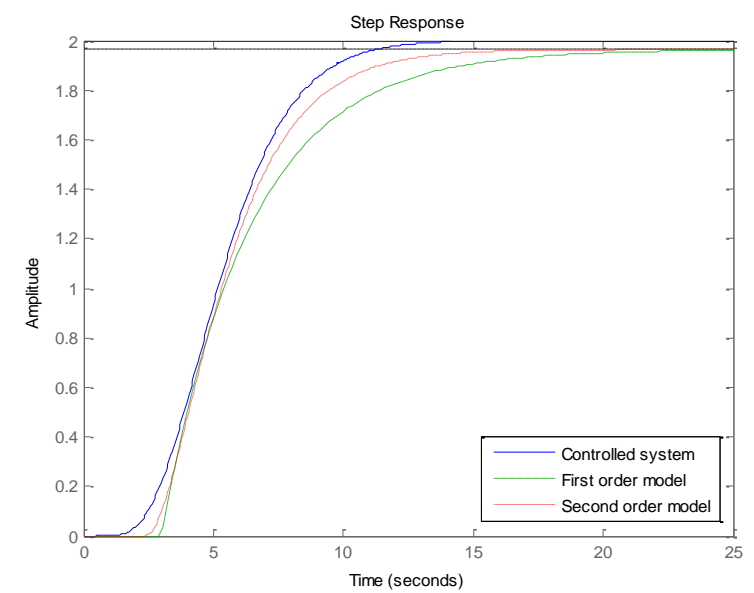

Fig. 5. Step responses of systems (8) and (9)

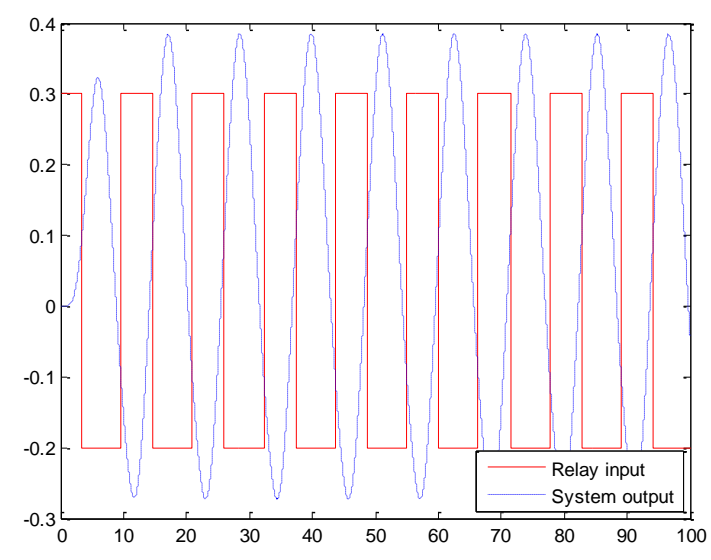

Fig. 6. Estimation experiment by asymmetrical relay 
A simpler class of controllers was derived in $R_{P S}$ representation with neglecting of time delay in the 1DOF configuration have the PI structure. Three responses for various tuning parameters $m>0$ are shown in Fig. 7 .

Generally, larger values of $m>0$ implicate larger overshoots and oscillations. As a consequence, for inaccurate relay identifications, lower values of $m>0$ can be recommended. The oscillatory responses of the controlled outputs are caused by neglecting of delay terms in (9), a deeper insight into this behavior can be found in [18], [19], [20], [21]. The PI controller for the default value $m=0.22$ gives the transfer function:

$$
C(s)=\frac{0.25 s+0.08}{s}=0.25\left(1+\frac{1}{3.125} \frac{1}{s}\right)
$$

Stability margins of the designed PI controllers with estimated control plant (8) are shown in Fig. 8. The open loop Nyquist plots also indicate that lower values of $m>0$ provide better stability margins, the distance from the critical point $(-1,0 j)$.

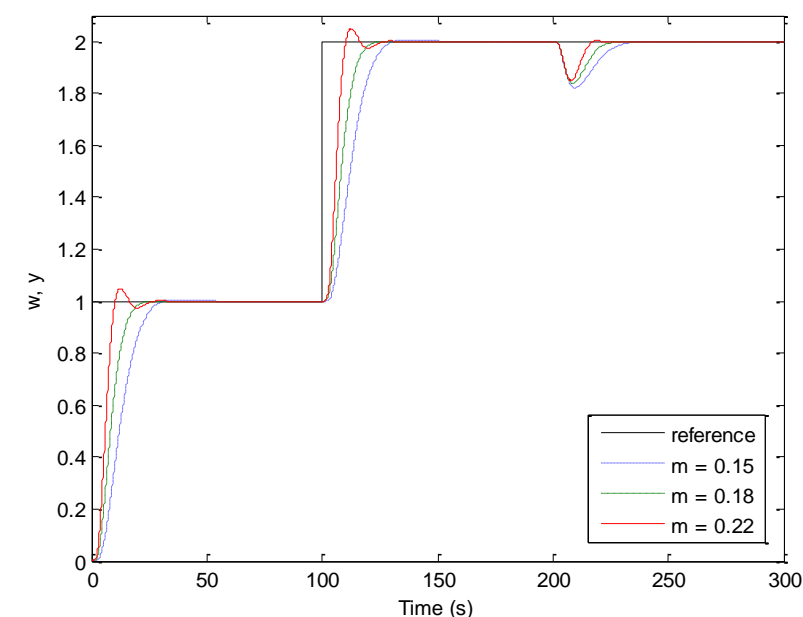

Fig. 7. Control responses $1 \mathrm{DOF}$ first order

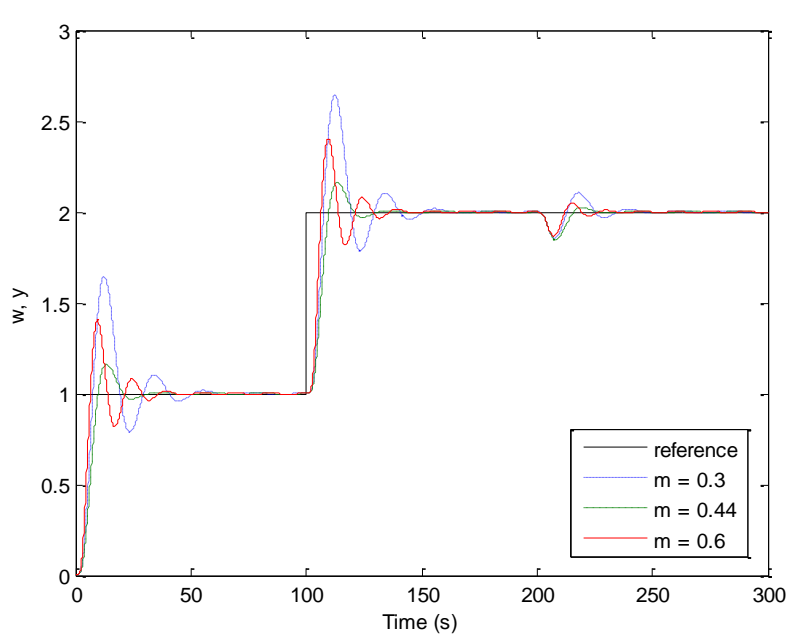

Fig. 9. Control responses 1DOF second order

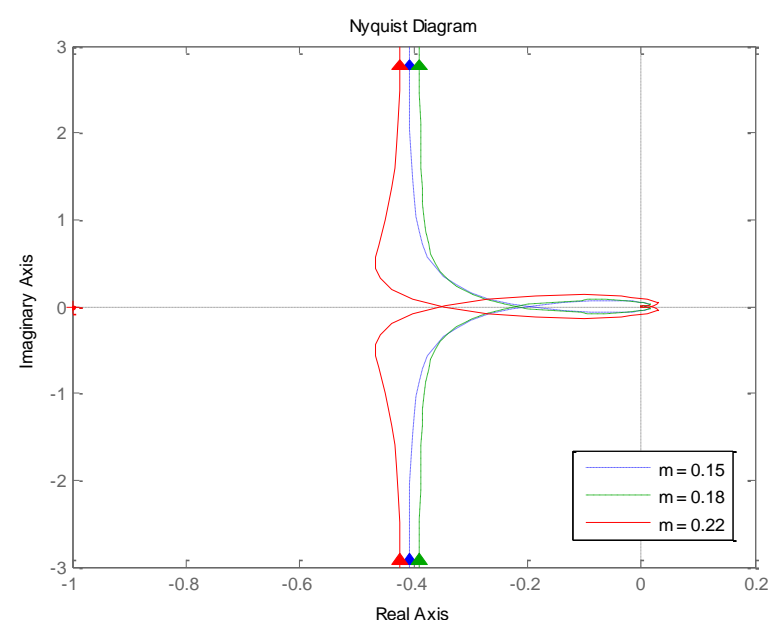

Fig. 8. Nyquist plot 1DOF first order

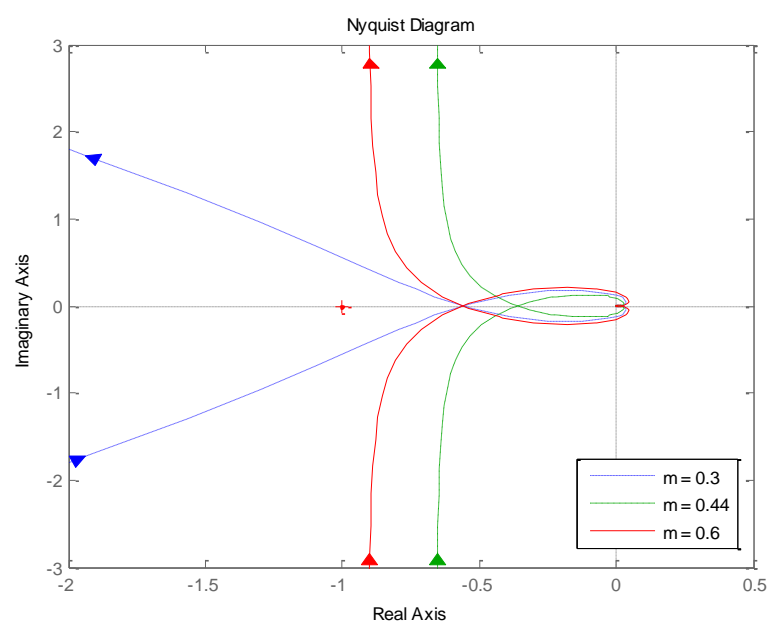

Fig. 10. Nyquist plot 1DOF second order

The second order identification and synthesis according to (9) in the 1DOF structure give a class of the PID controllers, for recommended value $m=0.44$ it takes the form:

$$
C(s)=\frac{0.33 s^{2}+0.35 s+0.09}{1.68 s^{2}+s}=\frac{s^{2}+1.06 s+0.27}{s(5.09 s+3.03)}
$$


The control responses of the controlled system (8) for three values of $m>0$ are shown in Fig. 9. Stability margins for these values are shown in Fig. 10. Both figures outline that the value $m=0.44$ achieves the best quality of control. Fig.10demonsrates the sensibility of the stability margin on the scalar parameter $m>0$. The best stability margin is achieved for the recommended value $m=0.4$, the other values gives worse behavior. Especially, for the lower value $m$ $=0.3$ the robustness of the closed loop is weak. This fact confirms that the methodology for choice of $m>0$ developed in [18] is correct.

Further improvement of the control behavior can be achieved by the change of the order of approximated model. The control system enables for FOPDT and SOPDT the Padé approximation of the time delay term. Naturally, the order of models as well as of the controller polynomials are higher and they are no more of PI and PID type. The FOPDT synthesis results in controller (for the default value $m=0.22$ ):

$$
\frac{Q}{P}=\frac{4.44 s^{2}+1.69 s+0.11}{18.33 s^{2}+s}=\frac{s^{2}+0.381 s+0.021}{s(4.128 s+0.225)}
$$

The second order estimation with Padé approximation gives the controller:

$$
\frac{Q}{P}=\frac{-0.08 s^{3}+0.04 s^{2}+0.12 s+0.04}{3.39 s^{3}+2.09 s^{2}+s}=\frac{-s^{3}+0.5 s^{2}+1.5 s+0.5}{s\left(42.375 s^{2}+26.125 s+12.5\right)}
$$

The resulting control responses of (8) by (12), (13) are shown in Fig. 11 and Fig. 12.

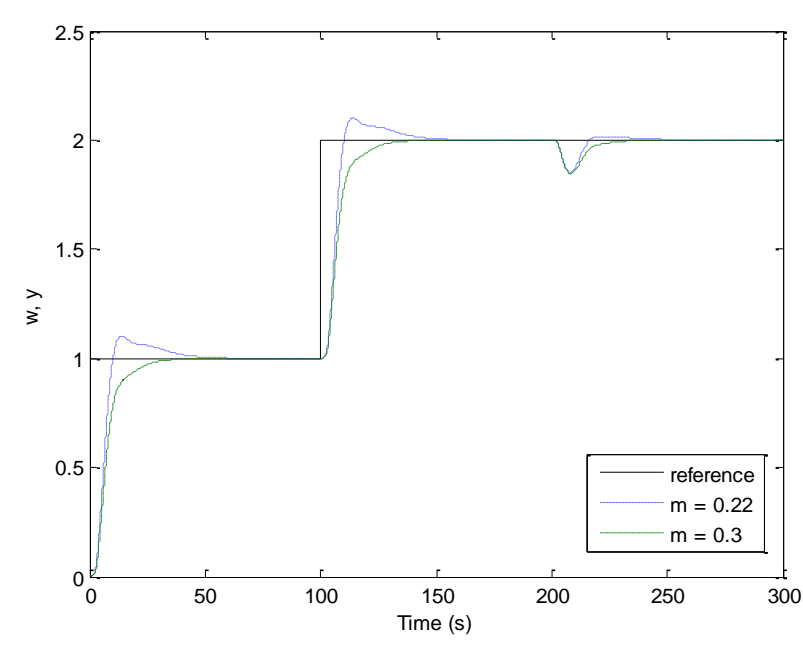

Fig. 11. Control responses (Padé approx.) first order

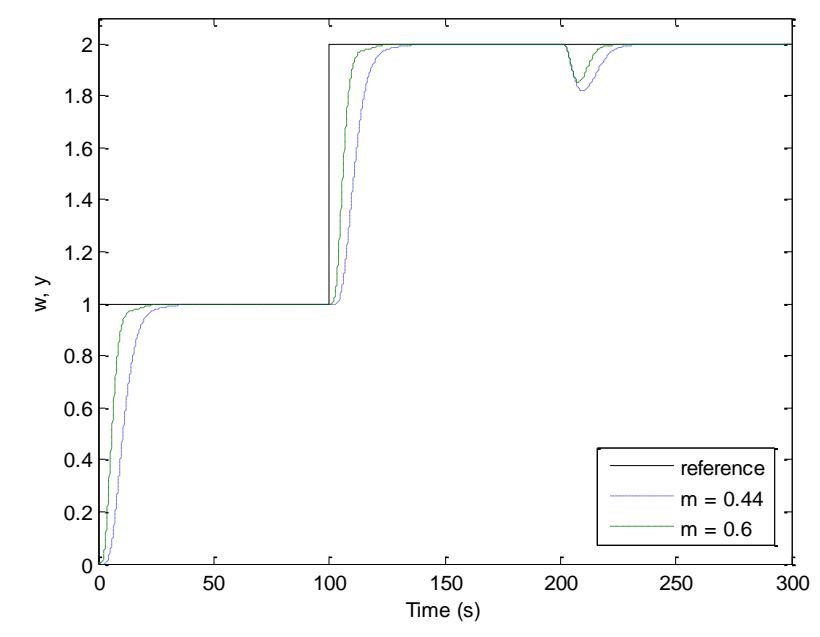

Fig. 12. Control responses (Padé approx.) second order

\section{Conclusion}

The main aim of the paper is to present a Matlab program system for controller design utilizing autotuning schemes. This contribution gives results from the long research of autotuning principles with a combination of relay feedback identification and a control design method. The estimation of first and second order transfer function parameters is performed from asymmetric limit cycle data, see [7], [8], [18], [19], [20]. The control synthesis is carried out through the solution of a linear diophantine equation according to [16], [17] in the ring of proper and stable rational functions $R_{P S}$. This approach defines a scalar tuning parameter which can be adjusted by various strategies ([4], [18], [20], [22]). A first order estimated model gives PI-like controllers while a second order model generates a class of PID ones. In both cases also the time delay term can be treated by the Padé approximation. Another approach for delay systems is presented in [19], [21]. A wide set of various controller then are derived. The Matlab program system was developed by this methodology for automatic design and simulation of feedback systems. An arbitrary higher controlled plant can be used for estimation and simulation. The program was developed in user friendly philosophy so that the user canot be familiar with the theory of algebraic control design. The resulting program system is suitable for control engineers as well as for students in automatic control.

The methodology is illustrated in an example of a high order system with a time delay term. A scalar tuning real parameter in all cases influences the control behavior as well as stability margin of designed control loops. Also, the influence of a scalar parameter is studied in the sense of stability margins. The deeper insight into various single input output systems for autotuning principles can be found in [7], [8], [16], [17], [18], [19], [20], [21]. 


\section{Acknowledgments}

This work was supported by the Ministry of Education, Youth and Sports of the Czech Republic within the National Sustainability Programme project No. LO1303 (MSMT-7778/2014) and by the European Regional Development Fund under the project CEBIA-Tech No. CZ.1.05/2.1.00/03.0089.

\section{References}

[1] Åström, K.J., \& Hägglund, T. (1984). Automatic tuning of simple regulators with specification on phase and amplitude margins. Automatica, Vol.20, pp.645-651.

[2] Åström K.J., \& Hägglund, T. (1995). PID Controllers: Theory, Design and Tuning. Research Triangle Park, NC: Instrumental Society of America.

[3] Garpinger, O, Hägglund, T. \& Åström K.J. (2012). Criteria and trade-offs in PID design, In: Preprints of IFAC Conferencieon Advancesin Control, Brescia (Italy).

[4] Gorez, R., \& Klán, P. (2000). Nonmodel-based explicit design relations for PID controllers, In: Preprints of IFAC Workshop PID'00, pp. 141-146.

[5] Hang, C.C., Åström, K.J. \& Wang, Q.C. (2002). Relay feedback auto-tuning of process controllers - a tutorial review, Journal of Process Control, Vol.12, No. 6, pp. 143-162.

[6] Kaya, I. \& Atherton, D.P. (2001). Parameter estimation from relay autotuning with asymmetric limit cycle data, Journal of Process Control, Vol. 11, No4, pp. 429-439.

[7] Korbel, J. \& Prokop, R. (2015). Accuracy of Relay Identification Depending on Relay Parameters. Procedia Engineering, No. 100, pp. 370-375. ISSN 1877-7058.

[8] Korbel, J.; Pekař, L. Prokop, Roman (2017). Delay Systems with Meromorphic Functions Design. In Annals of DAAAM International 2017, Volume 28. Vienna: DAAAM International Vienna, 2017, pp. 224-228. ISSN 23041382. ISBN 978-3-902734-14-3.

[9] Luyben, W.L. (2001). Getting more identification from relay-feedback tests. Ind. Eng.Chem. Res., Vol. 40, pp.4391-4402.

[10] Majhi, S. (2007). Relay based identification of processes with time delay. Journal of Process Control, Vol. 17, pp. 93-101.

[11] Majhi, S. \& Atherton, D.P. (1998). Autotuning and controller design for unstable time delay processes, In: Preprints of UKACC Conf an Control,pp. pp.769-774.

[12] Morilla, F., Gonzáles A. \& N. Duro (2000). Auto-tuning PID controllers in terms of relative damping, In: Preprints of IFAC Workshop PID’00, pp. 161-166.

[13] O`Dwyer, A. (2003). Handbook of PI and PID controller tuning rules. London: Imperial College Press.

[14] Panda, R.C., \& Yu, Ch.Ch. (2003). Analytical expressions for relay feedback responses. Journal of Process Control, Vol. 13, pp. 489-501.

[15] Pecharromán, R.R., \& Pagola, F.L. (2000). Control design for PID controllers auto-tuning based on improved identification, In: Preprints of IFAC Workshop PID’00, pp. 89-94.

[16] Prokop, R. \& Korbel, J . (2005). PI autotuners based on biased relay identification. In: Preprints of the $15^{\text {th }}$ IFAC World Congress, Prague.

[17] Prokop, R., Korbel, J. \& Prokopová, Z. (2010). Relay feedback autotuning - A polynomial approach, In: Preprints of 24th European Conf. on modelling and Simulation, Kuala Lumpur.

[18] Prokop, R., Korbel, J., \& Líška, O. (2011). A novel principle for relay-based autotuning. International Journal of Mathematical Models and Methods in Applied Science, Vol. 5, No. 7, s. 1180-1188.

[19] Prokop, R., Korbel, J., \& Pekař, L. (2015). Algebraic methods in autotuning design, Part I: Theory and design. In: Proc. of the $4^{\text {th }}$ CSOC Advances in Intelligent Systems and Computing. Heidelberg: Springer-Verlag Berlin, 2015, s. 67-78. ISSN 2194-5357. ISBN 978-3-319-18502-6

[20] Prokop, R., Korbel, J., \& Matušů, R. (2015). Algebraic methods in autotuning design, Part II: Implementation and simulation.In: Proc. of the $4^{\text {th }}$ CSOC Advances in Intelligent Systems and Computing. Heidelberg: SpringerVerlag Berlin, 2015, s. 79-89. ISSN 2194-5357. ISBN 978-3-319-18502-6.

[21] Prokop, R., Korbel, J. \& Pekař, L. (2016). Delay systems with meromorphic functions design. In The 12th IEEE International Conference on Control and Automation. New York, p. 443-448. ISSN 1948-3449. ISBN 978-1-50901738-6

[22] Thyagarajan, T. \& Yu, Ch.Ch. (2002). Improved autotuning using shape factor from relay feedback, In: Preprints of IFAC World Congres.

[23] Vítečková, M. \& Víteček, A. (2010). Plant identification by relay methods. In: Engineering the future (edited by L. Dudas). Sciyo, Rijeka, pp. 242-256.

[24] Yu, Ch.Ch. (1999). Autotuning of PID Controllers. Springer, London. 